Théologiques

Théologiques

\title{
Origines et actualité de la laïcité
}

Lecture socio-théologique

\section{Solange Lefebvre}

Volume 6, numéro 1, mars 1998

Autres regards sur la laïcité

URI : https://id.erudit.org/iderudit/024956ar

DOI : https://doi.org/10.7202/024956ar

Aller au sommaire du numéro

\section{Éditeur(s)}

Faculté de théologie de l'Université de Montréal

\section{ISSN}

1188-7109 (imprimé)

1492-1413 (numérique)

Découvrir la revue

\section{Citer cet article}

Lefebvre, S. (1998). Origines et actualité de la laïcité : lecture socio-théologique. Théologiques, 6(1), 63-79. https://doi.org/10.7202/024956ar
Résumé de l'article

La réflexion théologique est intriguée par le mot « laïcité » et les échos qu'il éveille : plus de cent ans après l'élaboration du projet de laïcité en France, qui rendait la raison souveraine et !'émancipait d'une autorité transcendante, nous nous trouvons toujours renvoyés, partout en Occident, d'une certaine manière, aux tensions historiques entre clercs et laïcs autour de la domination de la sphère spirituelle. Présentement, le phénomène de désinstitutionnalisation de la religion évoque ces tensions, c'est-à-dire que l'individualisation contemporaine de la croyance origine pour une part de la subjectivisation chrétienne laïque de l'expérience religieuse qui s'est affirmée surtout à partir du Moyen Age. La référence aux clercs n'a pas pour autant été éliminée, même si elle a muté, ce qui ressort dans les nouveaux rapports des modernes aux traditions, et plus spécifiquement aux institutions religieuses. Cette mutation renvoie à une révision fondamentale moderne de la dichotomie entre sacré et profane. Même s'il fut forgé pour exprimer tout autre chose au XIXe siècle, le mot « laïcité " revêt une prégnance théologique profonde. 
Théologiques 6/1 (1998) 63-79.

\title{
Origines et actualité de la laïcité
}

\section{Lecture socio-théologique}

\author{
Solange LEFEBVRE \\ professeure adjointe \\ Faculté de théologie \\ Université de Montréal
}

\section{RÉSUMÉ}

La réflexion théologique est intriguée par le mot "laïcité " et les échos qu'il éveille : plus de cent ans après l'élaboration du projet de laïcité en France, qui rendait la raison souveraine et l'émancipait d'une autorité transcendante, nous nous trouvons toujours renvoyés, partout en Occident, d'une certaine manière, aux tensions historiques entre clercs et laïcs autour de la domination de la sphère spirituelle. Présentement, le phénomène de désinstitutionnalisation de la religion évoque ces tensions, c'est-à-dire que l'individualisation contemporaine de la croyance origine pour une part de la subjectivisation chrétienne laïque de l'expérience religieuse qui s'est affirmée surtout à partir du Moyen Âge. La référence aux clercs n'a pas pour autant été éliminée, même si elle a muté, ce qui ressort dans les nouveaux rapports des modernes aux traditions, et plus spécifiquement aux institutions religieuses. Cette mutation renvoie à une révision fondamentale moderne de la dichotomie entre sacré et profane. Même s'il fut forgé pour exprimer tout autre chose au $\mathrm{XIX}^{\mathrm{e}}$ siècle, le mot "laïcité » revêt une prégnance théologique profonde.

Theological reflection has been intrigued by the word "laicity * and the reverberations that it arouses. More than one hundred years after the elaboration of the "project of the laicity * in France, which rendered reason sovereign by freeing it from a transcendental authority, we find ourselves returning, especially in the West, to historical tensions between the clergy and the laicity with respect to domination in the spiritual sphere. Presently, the phenomenon of the de-

L'auteure tient à remercier Robert David, Guy Durand et Guy Lapointe pour leurs remarques judicieuces, durant l'étape de rédaction finale. 
institutionalisation of religion evokes these tensions, that is, that contemporary individualisation of belief arises on the one hand, by the subjectivisation of lay Christian religious experience that was affirmed, for the most part since the Middle Ages. Reference to clergy had not been once and for all eliminated, even though it had undergone changes with respect to the new relationships between moderns and their traditions, and more specifically with respect to their religious institutions. This mutation refers to a fundamental modern revision of the dichotomy between the sacred and the profane. Even if it was conceived to express something completely different in the 19th century, the word "laicity * garners a deep theological meaning.

L'ardeur que met le monde moderne à revendiquer sa qualité de "laïque " montre bien qu'il n'a tien oublié de l'origine et de la portée du mot.

Georges de LAGARDE

Pour le théologien, le mot «laïcité » raisonne étrangement aux oreilles. Il définit d'abord une idéologie française, ainsi que l'indique Jacques Zylberberg :

Comme doctrine, comme idéologie, la laïcité est un phénomène singulièrement lié à l'histoire républicaine française, à la construction d'une gouverne atypique dans le monde occidental, dans ses relations avec la société civile, son ordonnancement centralisé des reproductions culturelles, sociales et économiques et sa production unitaire d'une citoyenneté informée par un projet rationaliste et républicain hégémonique dans la Cité (ZYLBERBERG 1995 : 37).

Au sens plus large, retenu par les dictionnaires usuels, il signifie "le principe de séparation entre l'Église et l'État, l'État n'exerçant aucun pouvoir religieux et les Églises aucun pouvoir politique : "La laïcité, c'est-àdire l'État neutre entre les religions» (RENANT)» (Petit Robert).

Pourtant, le terme "laïcité " évoque aussi en lui-même un pôle essentiel du christianisme, le pôle "laïc». Aux plans étymologique et historique raisonne en lui l'écho des tensions anciennes entre les laïcs et les clercs à l'intérieur du catholicisme, avant que ne s'opère la distinction moderne entre l'Église et l'État ${ }^{1}$. N'associe-t-on pas traditionnellement les

1 Déjà le roi saint Louis remettait en cause la théocratie, en distinguant les responsabilités juridiques. Cette distinction fut ensuite inscrite dans le droit par son petit-fils Philippe le Bel. "L'émancipation juridique devançait de loin celle des instances politiques et les luttes d'influence entre clercs et laiqques ne datent pas du XIX ${ }^{\mathrm{e}}$ siècle " (DEFOIS $1995: 28$ ). 
esprits läques à des positions anti-cléricales? La spécificité de l'idéologie laïque française, telle qu'elle fut élaborée au XIX ${ }^{\mathrm{e}}$ siècle, de même que l'idée de neutralité, tendent à occulter les traces de ces origines. L'avantlaïcité est vu alors comme un passé en ruines, et l'on dénie ce qui dure et se transmet, se remémore et se transfert.

Un autre concept signifie l'émancipation des sociétés de la tutelle religieuse, celui de sécularisation. Il a reçu d'innombrables définitions, dont voici quelques traits essentiels : la sécularisation représente un changement social, culturel, politique et religieux majeur : la centralité de la religion est remise en question. Le christianisme cesse d'être la source d'une vision du monde commune à tous, une organisation dominante définissant les tôles et les statuts sociaux qui structurent l'existence, et l'instance de régulation des croyances. L'une des principales conséquences de la sécularisation apparaît dans le fait qu'une société peut désormais se dispenser de la légitimation religieuse pour la définition de ses valeurs. Désormais, l'être humain gère son monde, vit et agit sans a-priori religieux; la fonction de la religion devient plus diffuse, même si elle subsiste (LEFEBVRE 1993 : 201). Dans ce qui suit, nous attirons davantage l'attention sur une définition théologique positive de la sécularisation, empruntée aux théologies de la sécularisation des années 1960, par exemple chez Harvey Cox (1968): le dépassement moderne de la dichotomie entre le sacré et le profane.

Par ailleurs, curieux revers de l'histoire, les analyses sociologiques les plus récentes du rapport entre institutions religieuses et sociétés occidentales, cessent d'accorder une importance dominante à la thèse de la « sécularisation" de ces sociétés, en tant qu'affaiblissement de l'influence


concerne la désinstitutionnalisation de la religion et ses conséquences pour les institutions de religions historiques, alors que l'individualisation de la croyance s'accentue (HERVIEU-LÉGER 1993). Ceci va de pair avec la pluralité des religions.

Luc Ferry, par exemple, débat de la "fin du théologico-éthique ": "Depuis Nietzsche, voire avec les « Lumières » déjà et leur critique de la superstition, nombre d'analyses ont considéré la naissance de l'univers démocratique comme l'effet d'une rupture avec la religion. "Mort de Dieu ", « désenchantement du monde " (Weber, Gauchet), fin du "théologico-politique " (Carl Schmitt), "sécularisation", " laïcisation " : plus ou moins contrôlées et controversées, ces expressions symbolisent aujourd'hui les multiples interprétations d'une même réalité : l'avènement d'un univers laïque au sein duquel la croyance en l'existence d'un Dieu ne structure plus notre espace politique » (FERRY $1996: 37$ ). La religion serait devenue affaire privée. 
Ces récents développements semblent donner raison au jeune Karl Marx qui pressentait ceci : "La religion ne se déploie dans son universalité pratique que là où n'existe pas de religion privilégiée " (1969: 142). Il répondait ainsi à Bauer qui estimait qu' « il n'y a pas de religion s'il n'y a plus de religion privilégiée ${ }^{3}$ ". La prolifération des religions ne se réaliserait que dans les sociétés qui ont cessé d'être religieuses. Le phénomène de prolifération des croyances n'est donc pas étranger au mouvement initié par la laïcité. Mais, en même temps que la subjectivisation des croyances, subsiste un attachement à la tradition religieuse, tant chez les juifs et les catholiques que chez les musulmans, - moins chez les protestants -, notamment pour des raisons de sauvegarde de l'identité culturelle.

Notre hypothèse est la suivante : pour une part, ce développement de la distinction entre croyance et institution apparaît s'inscrire dans la foulée même des rapports historiques entre clercs et laïcs. Selon les termes d'une théologie classique, repris notamment par l'historien Georges de Lagarde (1956), ces rapports se sont noués durant plusieurs siècles sur la base d'une dispute autour de la domination de la "sphère spirituelle" (distinguée de la sphère temporelle ${ }^{4}$ ). Il nous semble déceler encore aujourd'hui des traces de cet ancien conflit. Ainsi l'école, par exemple, pour bien des parents, s'avère-t-elle un lieu propice à l'éducation religieuse de leurs enfants, car elle se situe à distance des Églises. Des analyses des nouveaux courants religieux observent que les croyances où chacun s'institue comme son propre guide, touchent particulièrement les classes moyennes de professionnels qui ont échoué dans leurs aspirations à atteindre les échelons supérieurs de la société. Et ceci, sans parler des sempiternelles tensions entre clercs et laïcs dans l'Église catholique. Par ailleurs, notons que là où la référence aux clercs s'est effacée, dans certaines Églises protestantes, l'attachement à l'institution s'est aussi affaibli. Ces dernières sont d'ailleurs les plus à l'aise avec les références à la laïcité et à la sécularisation, ayant construit plusieurs discours théologiques à partir d'elles (COX 1968; REYMOND 1990).

Certes, notre but n'est pas de récupérer la référence à la laïcité idéologique française, mais de montrer que perdure dans nos débats actuels sur la laïcité, un rapport à la religion formé dans la matrice chrétienne. Le mot " laïcité " revêt à cet égard une prégnance théologique, même s'il

3 Commentant cette affirmation de Marx, Thériault écrit : "La subjectivité abstraite qui sert de fondement aux démocraties modernes active les prétentions des intersubjectivités particulières à occuper le lieu du pouvoir " (THÉRIAULT 1996:172).

4 La sphère spirituelle indique tout ce qui a trait au domaine ecclésiastique, par exemple le pouvoir sacerdotal, la régulation des croyances et de la morale. 
fut forgé pour exprimer tout autre chose au XIXe siècle. Pour vérifier cela, il importe de se mettre d'abord en quête de la mémoire du mot. Suivra une réflexion sur le rapport entre la laïcité et la désinstitutionnalisation de la religion. Une troisième et dernière partie réfléchira sur les transactions entre religions et sociétés laïques, qui illustrent cette désinstitutionnalisation, en même temps que ses limites. En définitive, la référence aux " clercs » n'aura pas été éliminée, mais elle aura muté.

\section{1. À l'origine de la laïcité : un dualisme structurel}

Le couple clerc-laïc renvoie au dualisme structurel qu'a instauré le catholicisme entre réalités spirituelles et réalités temporelles. Il en va de même pour le couple religieux-séculier. Dans le premier cas on réfère au statut relié à une fonction ecclésiastique et au pouvoir exercé dans l'Église, dans le second on réfère à l'état de vie en relation avec la poursuite de l'idéal de la perfection évangélique. Dans les deux cas, le second terme, soit les termes "laïc " et " séculier", se voit grevé d'un coefficient négatif, parce qu'il se trouve lié à la subordination du domaine temporel par le domaine spirituel.

Le couple clerc-laïc a une signification davantage politique : le latin laicus, qui vient du grec $\lambda a i$ kó $\xi$, signifie «du peuple». Le terme s'oppose à clericus ( $\mathrm{X}$ e siècle) signifiant "du clergé ", c'est-à-dire quelqu'un qui exerce des fonctions ecclésiastiques et, plus tardivement, à partir du XVe siècle, le lettré ou le savant. Le couple religieux-séculier revêt un sens qui, d'une certaine manière, est plus fondamental. Il réfère en effet à la séparation entre le sacré et le profane. Il permet de s'interroger sur la conception chrétienne du monde. Harvey Cox, dans La cité séculière, avait signalé une subtilité étymologique rattachée au mot "siècle ", qui a beaucoup de conséquences sur cette conception. Le terme "monde", observe$\mathrm{t}$-il, réfère aussi bien au latin mundus, qui évoque le cosmos grec, c'est-àdire une vision spatiale et plutôt statique du monde, qu'au latin saeculum, qui se rattache à l'idée de temps.

Et il se trouve que le monde comme sæculum, "siècle " nous rapproche davantage de la conception hébraïque du monde comme histoire. Pourtant, dans la tradition chrétienne, la conception du monde comme espace a peu à peu dominé, favorisant le départage spatial entre sacré et profane. Si bien que même le terme "siècle" a pris le sens d'espace profane. Ce sont les recherches bibliques du $\mathrm{XX}^{\mathrm{e}}$ siècle qui ont permis d'attirer l'attention sur cette distinction fondamentale (COX 1968). Cox avait fait ces observations à partir de sa réflexion sur le sens théologique de la sécularisation. Au-delà des visions assez négatives de celle-ci comme effacement de la religion dans le monde, Cox y voyait une révision salu- 
taire de la séparation entre sacré et profane. Le sens du "siècle" ou de son dérivé, la "sécularité ", se voit alors refondé dans l'historicité ${ }^{5}$.

La dichotomie entre le sacré et le profane s'est répercutée peu à peu aussi sur les rapports entre clercs et laïcs, entre Église et État. La vision dualiste apparaît au $\mathrm{XI}^{\mathrm{e}}$ siècle, avec la Réforme grégorienne. Apparaissent alors les deux pouvoirs: une distinction entre l'Église divine et cléricale, et l'État, temporel et laïc. L'opposition cléricale à l'intrusion laïque se fait radicale. Les revendications d'autonomie de l'Église, de séparation entre clercs et laïcs, entre Sacerdoce et tègne, viennent en premier des clercs eux-mêmes qui, à la fin du $\mathrm{XI}^{\mathrm{e}}$ siècle et au début du $\mathrm{XII}$ e siècle, aspirent à la "liberté ". Cette séparation renvoie peu à peu les laïcs à leur identité propre et bon nombre d'entre eux en viendront, finalement, à s'opposer aux clercs. Ceci fait dire à Jacques Chiffoleau qu'à certains égards, "les laïcs, au sens théologico-politique du terme - et ce qu'on appelle aussi, très maladroitement, la "laicisation », et même la «laïcité " -, sortent donc tout droit de la réforme grégorienne " (1992: $\left.55^{6}\right)$.

Durant le Bas Moyen Âge, le terme laicus prend un sens fortement juridique et institutionnel (GILCHRIST 1967). Comme antonyme de clerus, le mot indique une subordination, "celui sous l'autorité de", et réfère à quelqu'un qui n'appartient pas au groupe de ceux qui exercent une autorité sacrée. Les grands auteurs de l'époque n'accordent que peu d'intérêt aux laïcs, si ce n'est pour mentionner leurs excès et leur subordination aux clercs.

De 1300 à la fin du Moyen Âge, les finances papales s'avèrent une source de dissension, et provoquent en partie l'émergence du sentiment anti-clérical. Avant 1300, on trouvait des clercs et des laïcs dans les deux camps. Le système binaire fonctionnait assez bien, jusqu'à ce que surviennent la Renaissance et la révolution économique qui créent un nouveau type de laïc : l'humaniste et le citoyen. La distance entre eux et le

5

$\mathrm{Au} \mathrm{XX}$ siècle, les cultures anglo-saxonne et germanique ont davantage réfléchi sur ce sens de la sécularité et de son dérivé, la sécularisation, alors que les cultures française et latine font davantage référence à la catégorie "laïc " et à ses dérivés (laïcisation, etc.). Ainsi dans la plupart des dictionnaires anglais, on traduit le terme "laïcité " par " secularity ", le terme " laïcisation " par « secularization». La langue allemande forge le terme "Verweltlichung " pour traduire "laïcisation", et elle traduit " laïcité " par " wellicher Character ". Tous ces termes renvoient au concept de monde.

6 Le Nouveau Testament offrait une tout autre vision: les termes grecs $\lambda a o^{\prime} s$ et $\lambda a i k o ́ \xi$ désignaient le peuple de Dieu, sans distinction, sinon celles entre les enseignants et les enseignés, que nous verrons plus loin avec Georges de Lagarde. 
clergé s'élargit et ils se dirigent ailleurs, notamment vers les hérésies médiévales des Waldenses et des Cathares. Celles-ci s'expriment à l'intérieur de mouvements laïcs, évangéliques et anticléricaux. Les Princes finissent par combattre ces hérésies, car elles prêchent une doctrine en faveur de l'individualisme laïc, qui menace tout autant leur régime théocratique que la structure hiérocratique de l'Église.

On peut dire que la période dont il vient d'être question, à partir de 1300, marque le début du déclin de l'autorité de l'Église sur les sociétés européennes. L'Église n'est pas préparée à l'émergence du concept de civis, ou "citoyen", qui remplace peu à peu le terme laicus. Ce changement indique une diminution des fonctions sociales de l'Église et interroge les distinctions traditionnelles. Mais surtout, l'Église échoue dans l'entreprise d'éducation des masses. Les premiers laïcs à contrôler leurs écoles sont les hérétiques.

Les hérétiques jouent un rôle très important par la suite. Aux XVIe et $\mathrm{XVII}{ }^{\mathrm{e}}$ siècles, les dissidents, les marginaux à l'intérieur du protestantisme, les « hérétiques » développent les idées modernes (TREVOR-ROPER 1972; DELUMEAU 1992). Plus exactement, il s'agit des hérésies reliées au calvinisme, et qui s'inspirent de la vision érasmienne tolérante, rationaliste, sceptique, mystique et libérale. Les contestataires de même souche qui se trouvent du côté du catholicisme n'ont pas une influence aussi grande, car l'Église catholique ne permet pas la création de partis ou de sectes : " en son sein, les divergences d'opinion doivent être exprimées de façon plus vague, en des «mouvements » (TREVOR-ROPER 1972 : 270).

Les origines du terme "laïcité " se trouvent dans la lutte pour le pouvoir spirituel en France. Introduisant une vision des rapports entre l'Église et le monde, telle qu'elle est comprise aux XVIII ${ }^{\mathrm{e}}$ et XIXe siècles, le terme prend néanmoins racine dans l'humanisme de la Renaissance et dans la période des Lumières. Vue sous l'angle de ces origines spirituelles, l'idéologie française de laïcité aura au fond ressaisi le sens politique des rapport entre laos et clericus, et mis radicalement fin à la subordination médiévale: "Aujourd'hui, la partie est jouée; l'humanité est nationale; le laïc a gagné ... Le clerc n'est pas seulement vaincu, il est assimilé ... Toute l'humanité est devenue laïque, y compris les clercs" (BENDA 1977 : 244).

\section{Au-delà du dualisme : l'esprit laïque ou la désinstitutionnalisation de la religion}

Les travaux de l'historien Georges de Lagarde éclairent les origines religieuses de la laïcité, que nous tentons jusqu'ici de mettre en évidence. Il parle quant à lui de "l'esprit laïque », et ses travaux s'arrêtent à la fin 
du Moyen Âge, avec l'étude de l'œuvre de Guillaume d'Ockham. Il n'aborde donc pas le concept de "laïcité », mais ses origines historiques. Voici comment il définit «l'esprit laïque »:

L'esprit laïque ne peut naître qu'au sein d'une société où la distinction entre clercs et laïques, entre spirituel et temporel, entre autorité laïque et autorité ecclésiastique est nettement marquée. Est-il, en effet, autre chose que l'expression passionnée de l'effort tenté par les laïques pour reprendre un domaine que l'Église leur conteste? (1956: 158).

Il retrace "La naissance de l'esprit laïque " au déclin du Moyen Âge, soit au XIII ${ }^{e}$ siècle, alors que la distinction entre les deux ordres de la société chrétienne est très nette. C'est à ce moment que se seraient affirmées certaines tendances fondamentales qui allaient former l'esprit laïque. Mais celui-ci trouvait déjà ses racines dans les premières Églises chrétiennes qui différenciaient le "peuple des fidèles enseignés" et les membres du sacerdoce. Cette distinction est favorisée par l'indépendance du clergé et suscite naturellement une opposition des laïques contre le clergé. De Lagarde caractérise ainsi cette première forme de l'esprit läque : les laïques cherchent à reprendre au sein de l'Église, le contrôle direct du spirituel.

La deuxième forme de l'esprit laïque relève de la seconde distinction instaurée par le christianisme, entre le pouvoir spirituel et le pouvoir temporel, entre l'Église et l'État. Elle surgit lorsque la société civile revendique "l'intégralité » de ses droits de laïque sur l'ordre social et politique.

Le troisième moment, moderne, ressaisit ces deux dimensions, mais va plus loin, en remettant en cause le principe du partage. Le terme "laique " signifie alors la négation de tous les droits exercés depuis des siècles par l'Église et par le clergé dans la vie sociale. Et, aspect important pour ce qui concerne la dimension religieuse de la laïcité, de Lagarde observe que le mot "laïque " exprime désormais le désir de reconstruire le "spirituel " de la nation en dehors de toute influence positive d'une Église ou d'un clergé : "On a voulu leur interdire l'accès du " chœur ». Ils resteront donc les "laïques", mais comme tels, ils auront leur enseignement, leur morale, leur vertu et, si le cœur leur en dit, leur religion * (1956:XI).

Certains courants protestants assumeront ce projet. Réfléchissant au fondement biblique des rapports entre catholicisme et protestantisme, et par-delà le couple clercs-laïcs, en perpétuelle bataille, Jean-Louis Leuba retrouve le paradigme biblique de la tension entre l'institution et l'événement. Ce paradigme aide à comprendre le sens théologique du conflit entre catholicisme et protestantisme, entre continuité-institution et 
nouveauté-charismes (LEUBA 1950). Leuba observe une différence entre l'apostolat du groupe des Douze et celui de Paul (LEUBA 1950:47-82). Le premier, institutionnel, relève d'un acte de fondation et d'élection du Christ (Mc 3,13-19; Lc 6,12-16); le second, spirituel, est inattendu et coïncide avec la rencontre du Ressuscité (Ac 9,1-19; 22,6-21). L'apostolicité de Paul est tout à fait originale. L'épître aux Galates en fonde l'« indépendance originelle » et l'extériorité : «Ils reconnaissaient que l'apostolat de Paul, né en dehors de leur collège, était aussi destiné par Dieu à accomplir en dehors d'eux la tâche qui était la leur. "

L'approche de Leuba déploie le schème dualiste de l'institution et de l'événement, du cadre historique et de l'initiative imprévue de Dieu, de l'interne et de l'externe. Dans les faits cependant, la complémentarité entre le pôle institutionnel et le pôle événementiel, entre l'Église institutionnelle judéo-chrétienne et l'Église spirituelle pagano-chrétienne, sera difficile à maintenir. L'hérésie marcionite, par exemple, concentre la dimension apostolique de l'Église dans la mission paulinienne. À l'inverse, avec la première épître de Clément s'est amorcé un mouvement d'oblitération de l'apostolat spirituel de Paul, mouvement contre lequel la Réforme s'opposera (LEUBA 1950: 84). Leuba montre toutefois que l'Église ne se limite pas à l'institution établie, il élargit les possibilités de son surgissement, en dehors et autrement.

Ces réflexions sur les origines de l'esprit laique s'accordent au sens plus récent d'une désinstitutionnalisation du religieux. En effet, après une phase de départage dualiste entre politique et religion, entre l'État et l'Église, entre la religion et l'a-religion, on se rend compte que le phénomène religieux entretient des transactions plus fines avec l'a-religion. Mais il tend de plus en plus à évacuer l'institution dans les quêtes de tout un chacun, selon une dynamique d'individualisation (CAMPICHE 1997). Pourtant, des rapports avec les institutions de religions historiques perdurent, et ils varient d'un pays à l'autre en Occident.

Le Groupe international d'analyse comparative de changement social travaille sur les sociétés française, allemande, américaine et québécoise. Il observe notamment que dans les quatre cas, l'Église influence toujours l'école, mais de manière différente. Il note une différence importante aux États-Unis, en comparaison avec les trois autres régions étudiées : l'absence de sécularisation et l'augmentation de la pratique religieuse, surtout dans les mouvements fondamentalistes. Dans les quatre cas, il observe l'attachement d'une majorité de gens à leur culture religieuse :

Les catholiques, les musulmans, les protestants et les juifs qui ne pratiquent pas ne refusent pas pour autant leur héritage culturel, ils le revendiquent même: la tradition religieuse familiale est un facteur 
primordial d'identité que chacun tient à entretenir. Les catholiques «identitaires» rejettent le magistère de l'Église mais se disent et se veulent de tradition catholique. Les musulmans qui ne pratiquent pas les rites religieux sont très attachés à la culture musulmane et en respectent les interdits. Il en va de même pour les juifs, et à un bien moindre degré, les protestants. Les juifs de New York qui ne vont jamais à la synagogue cotisent pour Israël et entretiennent leurs écoles pour que leurs enfants soient élevés dans leur culture traditionnelle (FORSÉ ET LANGLOIS 1995 : 27-32).

Ainsi, présentement, la demande d'institution du religieux va-t-elle de pair avec son individualisation; la demande de référence religieuse avec sa privatisation, son intériorisation (CAMPICHE 1997). Danièle HervieuLéger observe qu'avec l'effritement des cadres de la mémoire collective et donc, des structures de transmission, on assiste à cette désinstitutionnalisation du religieux. Et celle-ci, " qui trouve son achèvement dans l'univers culturel de la haute modernité, est, pour une part au moins, une retombée de la subjectivisation chrétienne de l'expérience religieuse ". Ce qui mute, de la sorte, c'est le statut de la tradition dans la modernité. Elle n'est plus considérée comme un "dépôt sacré mais comme un patrimoine éthico-culturel, comme un capital de mémoire et comme un réservoir de signes à la disposition de l'individu " qui les assimile à la vérité subjective de sa trajectoire croyante $(1993: 289)$.

C'est cela que semble viser Finkielkraut, lorsqu'il diagnostique une dégénérescence de la laïcité comme saine distinction entre État et religion, en profanation de l'espace religieux, alors que tout un chacun fait subir aux organisations religieuses une critique sociale pas toujours appropriée. De la sorte la société absorberait en elle-même l'instance sacrée. À la lumière de ce qui vient d'être dit, on peut retrouver l'ancienne tension entre laïcs et clercs qui se disputent l'espace sacré, et surtout un aboutissant de la subjectivisation religieuse (FINKIELKRAUT 1995).

Au XIX ${ }^{e}$ siècle, les protestants ont appuyé l'idéologie de laïcité, espérant renverser sa tendance de passer de l'anticlérialisme à l'antichristianisme. Leur option théologique se résume de la sorte: "le christianisme est laïc par essence et par principe, sinon il ne serait pas de Dieu» (REYMOND 1990: 154). Le rapport entre foi et institution se trouve au cœur de la réévaluation que fait Reymond de cette réponse de la théologie calviniste à la laïcité : il conclut qu'elle a échoué dans son pari d'éliminer peu à peu la dimension institutionnelle, au profit de la foi seule. Un autre auteur protestant, Olivier Abel, se tient quant à lui dans la plus pure tradition protestante laïque: "Le ridicule ou le scandale tient d'abord pour nous à ce curieux usage de la religion et du sacré, qui consiste à en faire une fonction d'identité. [...] Le sentiment chez les protestants est très 
répandu que l'on peut rester protestant ... sans appartenir à aucune Église " (ABEL 1992: 116-7). Abel trouve une certaine naïveté, tant chez les catholiques, les juifs que les musulmans, de tenir à la pérennité de leur tradition. Il soutient même que les protestants acceptent de disparaître.

Reymond nous apparaît plus réaliste. Il renvoie le retrait de l'institution à une perspective eschatologique :

Le projet d'une théologie laïque reste grand et convaincant par son courage d'avancer à découvert sur un glacis qui ne lui appartient pas, mais dont elle demeure persuadée qu'il ne saurait être étranger à Dieu. Qu'elle assume alors tout le paradoxe de cette situation : elle ne peut se permettre aucun angélisme et ne peut jouer sa carte que par le biais de médiations symboliques et institutionnelles" (REYMOND $1990: 165)$.

Ce court développement présente en quelque sorte un enjeu caché de la laïcité : la tension entre institution, foi chrétienne et croyances. Elle parcourt l'histoire biblique, chrétienne et moderne, et ne semble pas vouloir se résoudre aisément dans l'élimination d'un terme ou de l'autre. De manière pratique, nos sociétés en sont présentement à l'heure des transactions diverses entre les trois, du dedans de la société civile et séculière.

\section{Nouvelle transactions entre institutions religieuses et sociétés}

Il nous semble nécessaire d'expliciter ici ce que sont devenus les rapports entre les institutions de religions historiques et les sociétés laiqques, à travers une revue de la littérature récente sur la laïcité. L'idéologie française de laïcité essuie de fortes critiques provenant d'un peu partout, y compris de la France elle-même. Comme idéologie rationaliste, elle se rattache à la démocratie abstraite moderne, contestée de part en part, surtout parce qu'elle suscite ce dont elle se veut la négation: les enracinements communautaires, culturels et religieux. Le Juif, Shmuel Trigano, exprime cela par cette image forte : on aurait scindé l'humain et le citoyen. Le citoyen relèverait du sacré de l'État, transcendant l'humain, et l'humain se rattacherait au profane. Cet écart serait le terreau des totalitarismes, des entreprises totalisantes d'un collectif détaché des sujets. La laïcité, prise à partie, est celle qui a dérivé en "positivité normative qui n'émancipait que l'homme dans le Juif ", par la sacralisation de l'État laïc, rival des institutions religieuses (TRIGANO 1992).

Zylberberg passe en revue les modèles allemand, canadien, américain et britannique, pour conclure que ces États et leurs droits publics respectifs, ne connaissent pas la conception stricte de la laïcité, et entretien- 
nent divers rapports aux religions majoritaires et minoritaires. On trouve à cet égard trois champs de vérification : l'autonomie de l'État, sa capacité à assurer lui-même sa reproduction culturelle dans l'espace scolaire, et celle de maîtriser les événements ponctuant le cycle de vie (naissance, mariage, divorce et mort). "Cette laïcité minimale, juridique, explique Zylberberg, est inversement proportionnelle aux droits des Églises, à leurs capacités juridiques, à leurs ressources financières, mais aussi à leurs ressources symboliques" (1995: 38).

Balthasar se questionne sur la douceur de la laïcisation des institutions et des mœurs québécoises (1990). Il préfère le concept de sécularisation pour aborder la question en Amérique du Nord, caractérisée par le "consensus idéologique », à la différence de l'Europe, davantage marquée par les divisions idéologiques. De ce côté-ci de l'Amérique, on a évité le débat fortement idéologique de la laïcité. On ne rencontre pas au Québec de mouvements intégristes ou anticléricaux orchestrés, et il ne reste guère de traces de l'Ancien Régime, contrairement à l'Europe où les racines de ce dernier demeurent étonnamment vivaces. Balthasar attribue au "pragmatisme " des Québécois, à leur attitude de conciliation, la persistance de la confessionnalité scolaire ${ }^{7}$. Celle-ci s'avère en effet de plus en plus anachronique, surtout dans un contexte où les nouveaux arrivants se voient obligés de fréquenter les écoles françaises et catholiques.

Dans la même publication, portant sur le territoire nord-américain et distinguant entre les États-Unis, le Canada anglais et le Québec, on tente de discerner les signes de "l'évolution des idéaux de la laïcité ». On y trouve le constat d'un Québec modernisé mais consumériste et hédoniste, d'où le questionnement sur le vide moral laissé par le retrait des Églises (RIOUX 1990). La thèse du vide est reprise, d'une certaine manière, par Juteau et Laurin, à travers une lecture de l'histoire des institutions hospitalières au Québec: les religieuses avaient réussi à recomposer finement économie domestique et capitalisme, famille et bureaucratie. Depuis la prise en charge des hôpitaux par les technocrates de la Révolution tranquille, il y a certes productivité accrue, mais sur fond de démembrement des milieux de travail et de leurs bases socioculturelles : fi la vocation et la dignité humaine, place aux ambitions promotionnelles des nouveaux patrons et à la vision des travailleurs comme une marchandise (JUTEAU et LAURIN 1990: 49; LAURIN 1996). Et surtout, il se produit une marginalisation des dimensions charitables, affectives et humanistes à l'intérieur des rationalités gestionnaires.

7 Notons que Balthasar écrivait cet article en 1990. La confessionnalité scolaire fut récemment atténuée par les récentes lois. 
On peut dire que, globalement, les critiques adressées à la modernité et à la raison instrumentale profitent aux institutions religieuses traditionnelles occidentales et aux peuples non occidentaux en général très religieux. Illustrons cela par quatre exemples: 1. Les avancées de l'État de droit et du pluralisme permettent à tous les groupes, y compris religieux, de coexister et de transiger dans l'espace public; 2. L'atomisation et l'anomie sociale activent les requêtes de sens, d'identité et de communauté, aisément ressaisies par les institutions religieuses; 3. Au plan idéologique, les institutions religieuses profitent des échecs des sociétés actuelles, au plan de l'exclusion et de la permissivité, qu'elles sont bien équipées pour critiquer; 4 . La classe politique qui n'a guère de légitimité aux yeux du public voit présentement l'avantage de s'associer aux religions historiques (judaïsme, christianisme, islam) (ZYLBERBERG 1995 : 50-51).

En outre, de manière génétale, présentement, les institutions religieuses profitent d'une faille laissée béante par les sociétés laïques, celle du déficit de culture religieuse, voire même de l'absence de reconnaissance de la dimension religieuse de la vie humaine. Cette faille aurait plusieurs conséquences: la déculturation des générations montantes, incapables de déchiffrer des œuvres majeures occidentales à référence juive ou chrétienne, le développement de fondamentalismes religieux et de croyances magiques dans le terreau propice de la scission entre raison et foi (DEFOIS 1995 : 31; GEFFRÉ 1992; BERGERON 1998); l'affaissement de l'éthique de responsabilité et la crise d'altérité, diagnostiquée dans plusieurs champs (ABEL 1992; GRAND'MAISON 1992).

Entre les esprits laïques et les esprits religieux, les oppositions se sont apaisées, du moins en Occident chrétien. Désormais il leur faut bien coexister, cohabiter. Les critiques réciproques sont même bienvenues. En ce sens Paul Valadier parle d'un "procès mutuel» que s'adressent la société lä̈que et les institutions religieuses (1987). Et comme plusieurs approches religieuses et morales entrent en concurrence pour influencer l'espace public, sur lequel la raison, la science et la technocratie ne peuvent plus en toute légitimité exercer leur hégémonie, on prône donc, ici et là, en faveur du partenariat (DEFOIS; FIGUIER, BAUBÉROT ...).

Les transactions diverses, pratiquées et appelées pour ces multiples raisons, peuvent laisser insatisfaits. S'agit-il en effet de colmater les brèches d'une modernité läque incapable de se refonder sur une sagesse véritable, par le "rattrapage", mais cette fois-ci, des traditions? Peutêtre. Mais pourquoi pas, pour le moment, admettre qu'entre l'institution religieuse et la subjectivité croyante, par-delà la dénégation, une saine tension critique est possible? Très pragmatique, Harvey Cox avait bien saisi qu'il n'y avait rien de mal, au contraire, à ce que la religion circule 
dans la cité séculière, comme il faut bien à la religion un vis-à-vis laïque pertinent.

\section{Conclusion : entre la mémoire et l'oubli}

Bien qu'appartenant à des horizons théoriques distincts, les termes "laïcité " et "séculier ", ainsi que leurs dérivés ont en commun à la fois la provenance d'un noyau sacré et d'un certain "affaiblissement " de cette provenance. Nous avons vu en première partie que les changements historiques et la situation religieuse de l'être humain dans le monde, induits par le renforcement du pôle temporel, contribuent à réviser la dichotomie sacré-profane, non biblique. C'est ce que la réflexion théologique entend par «sécularisation». Et elle s'est attelée justement à revoir la vision dualiste, qui relève d'une certaine métaphysique, au profit d'une vision du monde où sociétés sécularisées, laïques, et histoire biblique, entrent davantage en coïncidence, à travers la dimension d'historicité.

L'esprit laịc a combattu en partie la spatialisation extrême du sacré et du profane qui s'est établie en contexte de chrétienté, et les autres départages qu'elle entraînait (pouvoir et degrés de perfection). Le vaste mouvement d'émancipation auquel on assiste depuis le $\mathrm{XI}^{\mathrm{e}}$ siècle, consiste pour une part à prendre acte du fait que la culture occidentale cherche à dépasser l'opposition sacré-profane qui n'est plus fonctionnelle. Il n'élimine pas pour autant la dynamique spatio-temporelle, l'institution au profit de la foi, mais il propose le paradigme encore plus fondamental de l'historicité.

Gianni Vattimo présente une autre définition de la sécularisation, fondamentale pour notre propos. Réfléchissant sur une renaissance actuelle de l'intérêt religieux, il entend lui aussi mettre en évidence que la sécularisation est "un trait constitutif d'une expérience religieuse authentique » : "Le mot désigne le processus de « dérive » qui détache la civilisation laïque moderne de ses origines sacrées [...] il s'agit d'un rapport de provenance à partir d'un noyau de sacré dont on s'est éloigné et qui reste cependant actif, fût-ce dans sa version "déchue ", déformée, réduite à des termes purement mondains» (VATTIMO 1998 : 36.9). Cette sécularisation positive signifie l'incarnation, c'est-à-dire l'affaiblissement, la liquidation du sacré violent (GIRARD) qui a façonné l'expérience religieuse de l'humanité. Vattimo rejoint en quelque sorte un trait de l'expérience religieuse contemporaine que nous avons mis en évidence, lorsqu'il se présente comme un «demi-croyant", avec une référence plus libre à l'institution catholique.

La révision profonde de la géographie sacré-profane opérée par l'esprit laïque contribue à parachever l'œuvre de réinterprétation de la 
divinité "naturelle", comme une altérité théiste, déterministe, absolue, terrifiante et punitive. Elle ouvre sur la liberté religieuse, certes, mais aussi, en régime chrétien, sur l'expérience d'un «christianisme plus amical ». Entre le maître, qui raisonne, et l'esclave, qui croit, selon le mot du laïque Condorcet, un autre type de citoyen croyant peut faire irruption. Il s'agit là d'un enjeu politico-théologique dont les institutions religieuses n'ont pas mesuré toute la portée : "Je ne vous appelle plus serviteurs, je vous appelle amis " (Jn 15,15).

\section{Bibliographie}

ABEL, Olivier. 1992. "L'éthique protestante et l'esprit de la démocratie ", dans FIGUIER : 1992, pp. 116-122.

BALTHASAR, Louis. 1990. "La laïcisation tranquille du Québec " dans La laïcité en Amérique du nord (LEMAIRE, Jacques, dir.) ..., pp. 31-42.

BAUBÉROT, Jean. 1990. Vers un nouveau pacte laïque?. Paris, Seuil, 267 p.

BENDA, Julien. 1977. La trahison des clercs. Paris, Grasset (Le Livre de Poche, 8309), $411 \mathrm{p}$.

BERGERON, Richard. 1998. Viure au risque des nouvelles religions. Chap. 2 : "Une menace à l'intelligence? Un conflit de rationalités?", Montréal, Médiaspaul, pp. 103-128.

BOST, Hubert (dir.). 1990. Genèse et enjeux de la laïcité. Christianisme et laïcité. Genève, Labor et Fides, $228 \mathrm{p}$.

CAMPICHE, Roland, J. 1997. Cultures jeunes et religions en Europe. Paris, Cerf, $386 \mathrm{p}$.

CHIFFOLEAU, Jacques. 1992. "L'invention médiévale", dans FIGUIER : 1992, pp. 52-63.

COX, Harvey. 1968. La cité séculière. Essai théologique sur la sécularisation et l'urbanisation. Paris, Casterman, $286 \mathrm{p}$.

DEFOIS, Gérard. 1995. "La laïcité, vue d'en face " dans Pouvoirs, La laïcité. Paris, Seuil, pp. 27-36.

DELUMEAU, Jean. 1992. «La Renaissance et ses ambiguiités», dans FIGUIER 1992, pp. 64-72.

FERRY, Luc. 1996. L'homme-Dieu ou le Sens de la vie. Paris, Grasset, 250 p.

FIGUIER, Richard. 1992. Dieux en sociétés. Le religieux et le politique. Paris, Autrement (série «Mutations "127), 181 p. 
FINKIELKRAUT, Alain. 1995. "La laïcité à l'épreuve du siècle ", dans Pouvoirs. La laïcité. Paris, Seuil, pp. 53-60.

FORSÉ, Michel et Simon LANGLOIS. 1995. Tendances comparées des sociétés post-industrielles. Paris, Puf, $270 \mathrm{p}$.

GEFFRÉ, Claude. 1992. "L'homme est une histoire sacrée", dans FIGUIER 1992 : pp. 105-115.

GILCHRIST, J. 1967. "Laity, vocation of dans New Catholic Encyclopedia, vol. 8. Washington, The Catholic University of America, pp. 330-335.

GRAND'MAISON, Jacques. 1992. Le drame spirituel des adolescents. Montréal, Fides (Cahiers d'études pastorales 10), 241 p.

JUTEAU, Danielle, et Nicole LAURIN. 1990. "La sécularisation et l'étatisation du secteur hospitalier au Québec, de 1960 à 1966 " dans (LEMAIRE, J., dir.) La laïcité en Amérique du Nord, pp. 43-55.

LAGARDE, Georges de. 1956. La naissance de l'esprit laïque au déclin du Moyen Âge. T. 1: Bilan du XIII e siècle. Louvain/Paris, Nauwelaerts/Béatrice-Nauwelaerts, 217 p.

LAURIN, Nicole. 1996. "Le projet nationaliste gestionnaire. De l'hôpital des religieuses au système hospitalier de l'État ", dans Les frontières de l'identité (ELBAZ, Michaël, dir.). Sainte-Foy/Paris, PUL/L'Harmattan, pp. 95-104.

LEFEBVRE, Solange. 1992. Sécularité et sécularisation, Ph.D.. Faculté de théologie, Université de Montréal, 619 p.

LEFEBVRE, Solange. 1993. "Perspectives fondamentales et rechercheaction ", dans La théologie pratique. Statut, méthodes, perspectives d'avenir (REYMOND, Bernard, et J.-M. SORDET, dir.). Paris, Beauchesne, pp. 201-209.

LEMAIRE, Jacques (dir.). 1990. La laïcité en Amérique du Nord. Bruxelles, Éditions de l'Université de Bruxelles (La pensée et les hommes 33/14), $137 \mathrm{p}$.

LEUBA, Jean-Louis. 1950. L'institution et l'Événement. Les deux modes de l'œuvre de Dieu selon le Nouveau Testament. Leur différence, leur unité. Paris, Delachaux et Niestlé, 141 p.

MARX, Karl et Friedrich ENGELS. 1969. La Sainte Famille ou critique de la critique critique. Contre Bruno Bauer et consorts. Paris, Éd. Sociales, $256 \mathrm{p}$. 
NIERMANN, Ernst. 1975. "Secularization. II. Laicism", dans Encyclopedia of theology. The Concise Sacramentum Mundi (RAHNER, Karl, dir.). Crossroad, New York, 1554-1563.

REYMOND, Bernard. 1990. «À la recherche d'une théologie laïque. Le pari des modernismes protestants d'expression française», dans Christianisme et modernité (DUCRET, Roland et all., dir.). Paris, Cerf, pp. 151-165.

RIOUX, Emmanuel, «Les Québécois et Québécoises et les grands problèmes moraux de notre temps", (LEMAIRE, J., dir). La laïcité en Amérique du nord, pp. 23-30.

THÉRIAULT, Jacques-Yvon. 1996. "Le démocratisme et le trouble identitaire ", dans Les frontières de l'identité (ELBAZ, Michaël, dir.). SainteFoy/Paris, PUL/L'Harmattan, pp. 165-179.

TREVOR-ROPER, H.R. 1972. De la Réforme aux Lumières. Paris, Gallimard; original anglais, 1956.

TRIGANO, Shmuel. 1992. "Judaïsme et modernité ", dans FIGUIER : 1992, pp. 123-137.

VALADIER, Paul. 1987. L'Église en procès. Catholicisme et société moderne. Paris, Calmann-Lévy, $241 \mathrm{p}$.

VATTIMO, Gianni. 1998. Espérer Croire. Paris, Seuil; original italien, 1996.

ZYLBERBERG, Jacques. 1995. "Laïcité, connais pas : Allemagne, Canada, États-Unis, Royaume-Uni " dans Pouvoirs. La laïcité. Paris, Seuil, pp. 37-52. 